\title{
Perfil Sociodemográfico e Nutricional de Pacientes Oncológicos em Terapia Nutricional Enteral
}

doi: https://doi.org/10.32635/2176-9745.RBC.2018v64n2.72

Sociodemographic and Nutritional Profile of Oncological Patients in Enteral Nutritional Therapy
Perfil Sociodemográfico y Nutricional de Pacientes Oncológicos en Terapia Nutricional Enteral

Michelle Martins Bortoletto'; lury Antônio de Souza'; Anna Marcella Neves Dias ${ }^{3}$; Nilva Maria de Almeida ${ }^{4}$; Elisa Grossi Mendonça ${ }^{5}$

Resumo

Introduçáo: Os pacientes oncológicos apresentam alto risco nutricional, sendo a desnutriçáo e a caquexia comumente observadas nesses indivíduos. A terapia nutricional enteral (TNE) é uma terapêutica que pode oferecer inúmeros benefícios. Objetivo: Identificar o perfil sociodemográfico e nutricional de pacientes oncológicos relacionando-os aos efeitos da TNE com estado nutricional e à evoluçáo clínica desses pacientes. Método: Estudo clínico retrospectivo com revisão de prontuários eletrônicos de pacientes admitidos, no período de setembro de 2016 a abril de 2017, em um hospital oncológico público e privado, no município de Juiz de Fora - MG. As análises estatísticas foram por meio de frequências e proporçôes e média e desvio-padráo a depender das variáveis, sendo $p<0,05$. Resultados: Entre os 96 prontuários analisados, $68,75 \%$ eram do gênero masculino, 54,16\% idosos, $52,1 \%$ ex-tabagistas, 39,6\% ex-etilistas e 46,9\% apresentavam câncer de cabeça e pescoço. Destes, 44,8\% apresentavam suspeita de desnutrição e 39,6\% estavam desnutridos. A média do índice de massa corporal (IMC) foi de 19,44 $4,83 \mathrm{~kg} / \mathrm{m}^{2}$ e o peso $52,91 \pm 12,97 \mathrm{~kg}$ antes da TNE, e $18,76 \pm 4,47 \mathrm{~kg} / \mathrm{m}^{2}$ e $51,01 \pm 12,69 \mathrm{~kg}$ após sete dias em TNE, não apresentando diferença significativa entre os valores obtidos antes e após a TNE. A classificação do percentual da perda de peso no início da TNE evidenciou 88,5\% com perda de peso grave e, após sete dias de terapia, houve uma redução nesse percentual para 46,9\%. Conclusóes: A maioria dos pacientes oncológicos apresenta um comprometimento progressivo do estado nutricional. A TNE contribuiu de modo positivo para o retardo da perda de peso desses pacientes.

Palavras-chave: Neoplasias; Desnutriçăo Proteico-Calórica; Nutriçăo Enteral.

\begin{abstract}
Introduction: Cancer patients are at high nutritional risk, with malnutrition and cachexia being commonly observed in these individuals. Enteral nutritional therapy (ENT) is a therapy that can offer numerous benefits for these. Objective: To identify the sociodemographic and nutritional profile of cancer patients, relating to the effects of ENT with nutritional status and clinical evolution of these patients. Method: Retrospective clinical study with review of electronic medical records of patients admitted from September 2016 to April 2017 at a public and private oncology hospital in the city of Juiz de Fora - MG. Statistical analyzes were by means of frequencies and proportions and means and standard deviations depending on the variables, being $\mathrm{p}<0.05$. Results: Among 96 charts analyzed, $68.75 \%$ were males, $54.16 \%$ were elderly, $52.1 \%$ were former smokers, $39.6 \%$ were ex- and $46.9 \%$ had head and neck cancer. Of these, $44.8 \%$ were suspected of malnutrition and $39.6 \%$ were malnourished. The mean BMI was $19.44 \pm 4.83 \mathrm{~kg} / \mathrm{m}^{2}$ and the weight was $52.91 \pm 12.97 \mathrm{~kg}$ before the ENT and $18.76 \pm 4.47 \mathrm{~kg} / \mathrm{m}^{2}$ and $51.01 \pm 12.69 \mathrm{~kg}$ after seven days in ENT, showing no significant difference between the values obtained before and after ENT. The classification of the percentage of weight loss at the beginning of ENT showed $88.5 \%$ with severe weight loss and after seven days of therapy there was a reduction in this percentage to $46.9 \%$. Conclusions: Most cancer patients present with progressive impairment of nutritional status. ENT was a therapy that contributed positively to the delay of weight loss in these patients.

Key words: Neoplasms; Protein-Calorie Malnutrition; Enteral Nutrition.
\end{abstract}

Resumen

Introducción: Los pacientes oncológicos presentan alto riesgo nutricional, siendo la desnutrición y la caquexia comúnmente observadas en esos individuos. La terapia nutricional enteral (TNE) es una terapéutica que puede ofrecer innumerables beneficios para estos. Objetivo: Identificar el perfil sociodemográfico y nutricional de pacientes oncológicos relacionando a los efectos de la TNE con estado nutricional y evolución clínica de esos pacientes. Método: Estudio clínico retrospectivo con revisión de prontuarios electrónicos de pacientes admitidos en el período de septiembre de 2016 a abril de 2017 en un hospital oncológico público y privado en el municipio de Juiz de Fora - MG. Los análisis estadísticos fueron a través de frecuencias y proporciones y medias y desviaciones estándar a depender de las variables, siendo p $<0,05$. Resultados: Entre 96 prontuarios analizados, el $68,75 \%$ eran del género masculino, $54,16 \%$ ancianos, $52,1 \%$ ex tabaquistas, el $39,6 \%$ etilistas y el $46,9 \%$ tenían cáncer de cabeza y cuello. De estos $44,8 \%$ presentaban sospechosos de desnutrición y 39,6\% desnutridos. El promedio de IMC fue de $19,44 \pm 4,83 \mathrm{~kg} / \mathrm{m}^{2}$ y el peso $52,91 \pm 12,97$ kilogramos antes de la TNE y 18,76 $\pm 4,47 \mathrm{~kg} / \mathrm{m}^{2}$ y 51,01 $\pm 12,69$ kilogramos después de siete días en TNE, no presentando diferencia significativa entre los valores obtenidos antes y después de la TNE. La clasificación del porcentaje de la pérdida de peso al inicio de la TNE evidenció un 88,5\% con pérdida de peso grave y después de siete días de terapia hubo una reducción en este porcentual para el 46,9\%. Conclusiones: La mayoría de los pacientes oncológicos presentan un compromiso progresivo del estado nutricional. La TNE fue una terapia que contribuyó positivamente al retraso de la pérdida de peso de estos pacientes.

Palabras clave: Neoplasias; Desnutrición Proteico-Calórica; Nutrición Enteral.

${ }^{1}$ Acadêmica de Nutrição da Universidade Presidente Antônio Carlos (Unipac), Campus II. Juiz de Fora (MG), Brasil. E-mail: michellebortoletto@yahoo.com.br. Orcid ID: https://orcid.org/0000-0003-4273-7345

${ }^{2}$ Acadêmico de Nutrição da Unipac, Campus II. Juiz de Fora (MG), Brasil. E-mail: iuryasouza@gmail.com. Orcid ID: https://orcid.org/0000-0003-3907-0439

${ }^{3}$ Fonoaudióloga. Docente da Faculdade de Nutrição da Unipac, Campus II. Juiz de Fora (MG), Brasil. E-mail: annamarcelladias@yahoo.com.br. Orcid ID: https:// orcid.org/0000-0001-9811-6738

${ }^{4}$ Nutricionista. Serviço de Nutrição e Dietética do Instituto Oncológico. Juiz de Fora (MG), Brasil. E-mail: nilvanut@bol.com.br. Orcid ID: https://orcid.org/0000-0001-5357-329X ${ }^{5}$ Nutricionista. Docente da Faculdade de Nutrição da Unipac, Campus II. Juiz de Fora (MG) e Campus Magnus Barbacena (MG), Brasil. E-mail: egmnutri07@yahoo. com.br. Orcid ID: https://orcid.org/0000-0003-2121-9557

Endereço para correspondência: Michelle Martins Bortoletto. Rua 21 de Abril, 117, apto. 403 - São Mateus. Juiz de Fora (MG), Brasil. CEP 36025-070. 


\section{INTRODUÇÃO}

O câncer é uma doença crônica multifatorial caracterizada pelo crescimento desordenado das células, as quais apresentam divisão rápida e se portam de forma agressiva e incontrolável, propiciando a formação de tumores malignos. Estes podem invadir tecidos e órgãos distantes ou vizinhos, ocasionando um processo denominado de metástase ${ }^{1}$.

Destaca-se como um recorrente problema de saúde pública e é uma das principais causas de óbitos no âmbito mundial, particularmente entre os países em desenvolvimento. Aproximadamente $80 \%$ a $90 \%$ dos casos de câncer estáo relacionados a fatores ambientais, tais como hábitos alimentares, medicamentos, ingestão de álcool, uso de tabaco, exposição à luz solar, sedentarismo, agentes químicos, infecçôes por vírus e o próprio envelhecimento. Além disso, essa patologia está indiretamente associada à baixa escolaridade e a desigualdades socioeconômicas ${ }^{2}$.

De acordo com o Instituto Nacional de Câncer José Alencar Gomes da Silva (INCA), os pacientes oncológicos apresentam alto risco nutricional por não se alimentarem de maneira suficiente para atingir suas necessidades energéticas, fato decorrente de uma série de fatores, como a doença de base, dores, náuseas, vômitos, ansiedade, inapetência, disfagia, depressão, incapacidade funcional, tratamentos agressivos e mesmo pelo ambiente hospitalar. Dessa forma, a desnutrição calórico-proteica e a caquexia são frequentemente observadas. Sendo assim, a anorexia é um sintoma frequentemente observado em pacientes oncológicos, que pode ocasionar quadros de desnutrição e evoluir para a caquexia ${ }^{3}$.

Diante desse cenário, a terapia nutricional enteral (TNE) é uma terapêutica que oferta benefícios e contribui para a recuperação e/ou manutenção do estado nutricional. A TNE, introduzida de forma precoce e adequada ao paciente, é um importante fator na promoção da saúde, diminuição do estresse fisiológico e manutenção da imunidade ${ }^{4}$.

Nesse contexto, o objetivo do presente estudo foi identificar o perfil sociodemográfico e nutricional de pacientes oncológicos e relacionar os efeitos da TNE no estado nutricional desses pacientes com a evolução clínica.

\section{MÉTODO}

Estudo de caráter observacional, retrospectivo, realizado em um hospital oncológico público e privado, situado no município de Juiz de Fora - MG, previamente aprovado pelo Comitê de Ética em Pesquisa da Universidade Presidente Antônio Carlos sob n 2.288.197.

A coleta de dados foi realizada em 96 prontuários eletrônicos constando os protocolos de atendimento nutricional de pacientes admitidos no período de setembro de 2016 a abril de 2017. Os critérios de inclusão foram os pacientes de ambos os gêneros, com idade superior a 18 anos e com permanência maior ou igual a sete dias em TNE. Foram excluídos os pacientes com retenção hídrica ou edema associado ao uso de corticoides, em razão da interferência desse quadro no peso e consequente influência na classificação do estado nutricional.

Os dados coletados foram: nome, gênero, idade, data de admissão, nível de escolaridade, profissão ou ocupação, tipo de convênio (SUS ou particular), tabagista ou extabagista, e etilista ou ex-etilista, diagnóstico ou hipótese diagnóstica, tratamento oncológico, comorbidades associadas, estatura $(\mathrm{m})$, peso atual $(\mathrm{kg})$, peso usual $(\mathrm{kg})$, índice de massa corporal (IMC) $\left(\mathrm{kg} / \mathrm{m}^{2}\right)$ e avaliação e classificação do risco nutricional pela avaliação subjetiva global produzida pelo próprio paciente (ASG-PPP), porcentagem de perda de peso e classificaçáo e presença ou ausência de caquexia mediante relato em prontuário segundo a classificação utilizada pelo hospital, sendo esta baseada no Consenso Internacional de Caquexia e no Consenso Brasileiro de Caquexia e Anorexia em Cuidados Paliativos, ambos de 2011. Em relação ao IMC, a sua classificaçáo se deu de acordo com os pontos de corte recomendados pela Organização Mundial da Saúde (OMS) para a população adulta e idosa.

Para as avaliaçóes antropométricas realizadas pelo setor de nutrição, foram utilizados os dados da avaliação que ocorreu na admissão do paciente e sete dias após a intervenção da equipe para TNE.

A análise estatística foi realizada por meio do software SPSS Statistics 17. A análise das variáveis categóricas (gênero, tipo de convênio: Sistema Único de Saúde (SUS) ou particular, nível de escolaridade, profissão ou ocupaçáo, tabagista ou ex-tabagista, etilista ou ex-etilista, diagnóstico, tratamento oncológico, comorbidades associadas, ASG-PPP, presença ou ausência de caquexia, classificação do IMC, classificaçáo do percentual da perda de peso) foi descrita por meio de frequência e proporçóes. Variáveis quantitativas (idade, estatura, IMC e peso atual no início e sete dias após TNE) foram descritas por meio da média e desvio-padrão. A significância das diferenças entre as médias antes e após o tratamento foi verificada com a aplicaçáo do Teste t para dados pareados. A significância das diferenças para as variáveis qualitativas foi verificada por meio da aplicação do Teste de Qui-quadrado de Mantel-Haenszel. Para todos os testes, considerou-se como diferença estatisticamente significativa valor de $\mathrm{p}<5 \%(\mathrm{p}<0,05)$.

\section{RESULTADOS}

O estudo compreendeu a análise de 96 prontuários eletrônicos de pacientes, sendo $68,7 \%$ do sexo masculino 
$(\mathrm{n}=66) ; 54,2 \%$ apresentavam idade igual ou superior a 60 anos ( $\mathrm{n}=52)$, com média de idade de $62,17 \pm 10,64$ anos (min. 40 - máx. 84 anos). De acordo com o convênio, o SUS correspondeu a $89,6 \%(\mathrm{n}=86)$ das internaçóes.

No que concerniu ao nível de escolaridade, a maior parte dos indivíduos apresentou o primeiro grau completo, totalizando $60,4 \%(n=58)$. A ocupaçáo mais prevalente foi a de aposentado $34,5 \%(\mathrm{n}=33)$, seguido de lavrador $10,4 \%(n=10)$, do lar $10,4 \%(n=10)$, comerciante $9,4 \%$ $(n=9)$, e outras $35,4 \%(n=34)$. Em relação ao tabagismo e etilismo, 52,1\% $(\mathrm{n}=50)$ eram ex-tabagistas, 5,2\% $(\mathrm{n}=5)$ tabagistas; 39,6\% ( $\mathrm{n}=38)$ ex-etilistas, 5,2\% ( $\mathrm{n}=5)$ etilistas. No que se refere ao diagnóstico médico, o câncer mais prevalente foi o de cabeça e pescoço, $46,9 \%$.

Ao relacionar o fumo e o álcool com o tipo de câncer, observou-se que os pacientes que eram ex-tabagistas apresentaram como diagnóstico o tumor de cabeça e pescoço $68 \%(\mathrm{n}=34)$; da mesma forma, constatou-se que $71,1 \%(\mathrm{n}=27)$ dos pacientes eram ex-etilistas e portadores do mesmo tipo de neoplasia. Quando se relacionou o gênero e o tipo de câncer, verificou-se que, entre os pacientes portadores de tumor de cabeça e pescoço, $54,5 \%$ $(n=36)$ eram do sexo masculino e apenas 30\% ( $n=9)$ eram do sexo feminino.

No que diz respeito ao tratamento oncológico, $87,5 \%$ $(\mathrm{n}=84)$ estavam em terapia antineoplásica, sendo mais frequente a quimioterapia $35,4 \%(n=34)$ seguida da radioterapia $29,2 \%(n=28)$. As comorbidades associadas ao câncer foram verificadas em 70,8\% ( $\mathrm{n}=68)$ dos pacientes, sendo prevalente a hipertensão arterial (HAS) com $42,7 \%(\mathrm{n}=41)$ e a HAS concomitante ao diabetes mellitus com $18,8 \%(\mathrm{n}=18)$.

De acordo com a classificação do risco nutricional por meio da ASG-PPP, foi constatado que $44,8 \%$ dos pacientes apresentavam suspeita de desnutrição e 39,6\% eram desnutridos, englobando moderadamente e gravemente desnutridos como demonstrado na Tabela 2 , sendo significativo $(\mathrm{p}<0,05)$ o percentual de desnutridos em relação às demais classificaçôes. Com relação à presença ou ausência de caquexia, foi verificado que $60,4 \%$ dos pacientes $(n=58)$ não apresentavam um quadro de caquexia, entretanto o percentual que evidencia o estado de extrema desnutrição, 39,6\% ( $n=38)$, é relevante.

Correlacionando a presença ou ausência de caquexia com o tipo de câncer, evidenciou-se que, entre os pacientes que apresentavam um quadro de extrema desnutrição, o maior número foi de portadores de câncer de cabeça e pescoço $(57,9 \%)$ quando comparados com os demais tipos de neoplasias, havendo assim diferença significativa $(\mathrm{p}<0,05)$.

A média de IMC antes da TNE foi $19,44 \pm 4,83$ $\mathrm{Kg} / \mathrm{m}^{2}$, e $18,76 \pm 4,47 \mathrm{~kg} / \mathrm{m}^{2}$ após sete dias de terapia, não sendo significativa $(\mathrm{p}>0,05)$ a correlação entre os parâmetros. A classificação do IMC (Tabela 1) mostrou $66,6 \%(n=64)$ de desnutrição na primeira avaliação e $69,7 \%(\mathrm{n}=67)$ sete dias após a TNE.

Tabela 1. Classificação do estado nutricional pelo índice de massa corporal antes e após 7 dias de terapia nutricional enteral

\begin{tabular}{l|c|c}
\hline Classificação & $\begin{array}{c}\text { Antes da terapia } \\
\text { nutricional } \\
\text { enteral N (\%) }\end{array}$ & $\begin{array}{c}\text { Após } 7 \text { dias } \\
\text { de terapia } \\
\text { nutricional } \\
\text { enteral N (\%) }\end{array}$ \\
\hline Eutrofia & $25(26,0)$ & $25(26,0)$ \\
\hline $\begin{array}{l}\text { Desnutrição } \\
\text { grau I }\end{array}$ & $39(40,6)$ & $41(42,7)$ \\
\hline $\begin{array}{l}\text { Desnutrição } \\
\text { grau II }\end{array}$ & $8(8,3)$ & $7(7,3)$ \\
\hline $\begin{array}{l}\text { Desnutrição } \\
\text { grau III }\end{array}$ & $17(17,3)$ & $19(19,8)$ \\
\hline $\begin{array}{l}\text { Obesidade } \\
\text { grau I }\end{array}$ & $7(7,3)$ & $4(4,2)$ \\
\hline Total & $96(100,0)$ & $96(100,0)$ \\
\hline
\end{tabular}

A respeito do peso antes da TNE e após sete dias do seu uso, os resultados obtidos neste trabalho mostraram uma média de 52,91 $\pm 12,97 \mathrm{Kg}$ antes da TNE, com peso mínimo de 20,21 Kg e máximo de $93,7 \mathrm{Kg}$, e uma redução

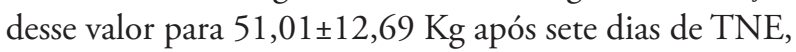
sendo o peso mínimo $20,21 \mathrm{Kg}$ e o máximo $86,6 \mathrm{Kg}$. Essa diminuição de peso encontrada não apresentou diferença significativa $(p>0,05)$ quando comparada ao peso inicial, antes da TNE. A classificação do percentual da perda de peso no início da TNE evidenciou que 95 pacientes $(98,9 \%)$ apesentavam perda de peso e, após sete dias de terapia, 46 pacientes $(47,9 \%)$ não tiveram perda de peso (Tabela 2). Dos pacientes que não apresentaram perda de peso, $26(27,1 \%)$ ganharam peso, $20(20,8 \%)$ mantiveram e $50(52,1 \%)$ continuaram a perder peso após a terapia.

Tabela 2. Classificação do percentual da perda de peso dos pacientes analisados antes e após 7 dias de terapia nutricional enteral

\begin{tabular}{l|c|c}
\hline Classificação & $\begin{array}{c}\text { Antes da terapia } \\
\text { nutricional } \\
\text { enteral N (\%) }\end{array}$ & $\begin{array}{c}\text { Após } 7 \text { dias } \\
\text { de terapia } \\
\text { nutricional } \\
\text { enteral N (\%) }\end{array}$ \\
\hline Perda grave & $85(88,5)$ & $45(46,9)$ \\
\hline $\begin{array}{l}\text { Não teve } \\
\text { perda }\end{array}$ & $1(1,0)$ & $46(47,9)$ \\
\hline $\begin{array}{l}\text { Perda } \\
\text { significativa }\end{array}$ & $10(10,4)$ & $5(5,2)$ \\
\hline Total & $96(100,0)$ & $96(100,0)$ \\
\hline
\end{tabular}




\section{DISCUSSÃO}

Os dados obtidos por meio dos prontuários analisados apontaram para uma predominância de pacientes do sexo masculino, idosos, com primeiro grau completo e aposentados. No que se refere ao tipo de convênio, o SUS representou $89,6 \%$ das internaçóes. Kolankiewicz et al. ${ }^{5}$ afirmaram, em seu estudo com pacientes oncológicos, que as doenças crônico-degenerativas possuem pior prognóstico quando associadas à baixa escolaridade e a desigualdades socioeconômicas.

A prevalência acentuada de homens portadores de neoplasias evidenciada neste estudo também foi observada nos estudos de Vieira et al. ${ }^{6}$, com pacientes de um hospital filantrópico da cidade de Cuiabá/MT, onde 79,2\% eram do sexo masculino.

A incidência dessa patologia no sexo masculino pode ser elucidada pela própria cultura masculina, na qual o autocuidado e a procura por serviços de saúde colocariam em risco sua masculinidade, sendo estas consideradas como atributos femininos. Do mesmo modo, a sua exposiçáo a alguns fatores de risco da doença, como tabagismo, álcool, sedentarismo, dieta desequilibrada e obesidade contribuem diretamente para sua ocorrência e mortalidade ${ }^{7}$.

A média de idade do presente estudo $(62,17 \pm 10,64$ anos) é semelhante à encontrada por Boventura et al. ${ }^{8}$, que verificaram o perfil de pacientes oncológicos de um hospital público do Estado de São Paulo, onde a idade média foi de 62 anos. O envelhecimento é um fator de risco para o desenvolvimento de alguns tipos de neoplasias. $\mathrm{O}$ aumento da incidência de câncer em idosos ocorre como resultado da exposição prolongada a carcinógenos, acúmulo de alteraçôes genéticas e disfunçôes imunes, o que pode justificar a maior prevalência de indivíduos nessa faixa etária, fazendo-se necessário reforçar estratégias de prevenção e controle da doença? .

No que se refere ao consumo de tabaco e álcool, os dados obtidos na presente pesquisa corroboram os encontrados nos estudos de Le Campion et al. ${ }^{10}$, ao analisarem prontuários de pacientes oncológicos de dois centros de referência em estomatologia do Estado de Alagoas, onde $91,7 \%$ já tinham sido expostos ao fumo e $77 \%$ ao álcool.

O tabagismo e o etilismo podem ser considerados os principais fatores de risco para o desenvolvimento de câncer, principalmente quando se trata de tumores primários de cabeça e pescoço, excetuando a tireoide. $\mathrm{O}$ cigarro contém substâncias que podem alterar o perfil molecular dos indivíduos e causar mutaçôes, e o álcool age como solvente aumentando a exposição da mucosa a agentes carcinogênicos, facilitando e elevando a sua absorção celular ${ }^{11}$.
Com relação aos tumores de maior prevalência encontrados no presente estudo, o de maior ocorrência foi o de cabeça e pescoço (46,9\%), resultado semelhante ao observado por Brito et al. ${ }^{12}$ no Sudoeste da Bahia, em um estudo com adultos e idosos assistidos em uma instituição de apoio ao paciente oncológico, onde $40,5 \%$ possuíam a mesma patologia.

A neoplasia de cabeça e pescoço é caracterizada pela agressividade local e pelo risco de desenvolvimento de tumores secundários, apresentando assim alta mortalidade e morbidade, e tem como principais fatores de risco o tabagismo e o etilismo ${ }^{13}$. O presente estudo revelou a predominância de neoplasias de cabeça e pescoço em indivíduos do sexo masculino e em pacientes que eram ex-tabagistas e ex-etilistas. Dessa forma, esses dados corroboram a incidência dessa patologia.

O tipo de tratamento antineoplásico que apresentou maior frequência na presente pesquisa foi a quimioterapia, seguida da radioterapia; entretanto, Medeiras et al. ${ }^{14}$, em um estudo com pacientes ambulatoriais submetidos a tratamento oncológico de um hospital da cidade de São José do Rio Preto - SP, encontraram a radioterapia concomitante à quimioterapia como mais prevalente. Isso pode ser explicado porque, no hospital onde foi realizado o presente estudo, a radioterapia faz parte do tratamento particular, e a maioria dos pacientes encontrava-se em tratamento pelo SUS.

De acordo com os dados obtidos no presente estudo, $70,8 \%$ dos pacientes apresentavam alguma comorbidade, sendo a HAS a mais prevalente. Esse dado também foi observado nos estudos de Azevedo e Bosco ${ }^{15}$, que relataram a presença de comorbidades em $55,0 \%$ dos pacientes analisados, sendo a HAS (25\%) a de maior proporção.

A hipertensão tem sido relatada como a comorbidade mais comumente encontrada em pacientes com neoplasias malignas. A sua associação com o uso de alguns agentes terapêuticos, principalmente os inibidores de angiogênese, pode acarretar a incidência ou o seu agravamento, e a sua ocorrência pode minimizar os benefícios do tratamento antineoplásico e reduzir a sobrevida, além de estar associada a um maior consumo de medicamentos que podem exercer impacto no apetite, na absorção, metabolismo e excreção de nutrientes ${ }^{16}$.

No que diz respeito à classificação do risco nutricional por meio da ASG-PPP, no presente estudo, a prevalência da desnutrição foi verificada em 39,6\% da amostra, sendo que, desta, $44,8 \%$ encontravam-se com suspeita de desnutriçáo. Esse resultado se assemelha a outros estudos que utilizaram o mesmo método. Pastore et al. ${ }^{17}$, em um estudo com 77 pacientes com câncer no trato gastrointestinal e pulmão, encontraram a desnutrição em $87,0 \%$ de sua amostra. 
De acordo com um estudo epidemiológico observacional realizado com 1.545 pacientes adultos selecionados, aleatoriamente, em centros de câncer na França, 30,9\% encontravam-se desnutridos, sendo $18,6 \%$ moderadamente desnutridos e $12,2 \%$ desnutridos graves. Relacionando a prevalência da desnutrição com o local do tumor e o tempo de hospitalização, os autores identificaram que o câncer de cabeça e pescoço e o tempo de internaçáo prolongado aumentam o risco de desnutriçãá ${ }^{18}$.

Cunha et al. ${ }^{19}$, ao relacionarem o motivo da internaçáo com a ocorrência de desnutrição em pacientes do Hospital das Clínicas de Ribeirão Preto - SP, constataram que os indivíduos com doenças neoplásicas apresentavam maior prevalência de desnutrição $(62,7 \%)$, sendo $49,5 \%$ desnutrição moderada e $12,7 \%$ desnutrição grave, quando comparados àqueles hospitalizados para tratamento de outras afecçóes. A desnutrição grave ocorreu principalmente em pacientes com neoplasia de cabeça e pescoço $(46,9 \%)$.

As diferenças encontradas nos percentuais podem ser explicadas pelas características dos pacientes de cada estudo, tais como faixa etária, tempo da doença, tipo de neoplasia e tratamento antineoplásico. Contudo, a ASG-PPP constitui-se como um método importante para avaliaçáo do risco nutricional, uma vez que é capaz de prenunciar complicaçôes, mortalidade e tempo de internação. Nesse contexto, o acompanhamento nutricional precoce e contínuo é de suma importância na tentativa de reverter ou melhorar esse quadro ${ }^{20}$.

A caquexia tumoral é a manifestação máxima da desnutrição em pacientes oncológicos, sendo responsável diretamente por um terço das mortes dos pacientes com câncer. Ela tem como características marcantes a perda de massa muscular, com ou sem perda de tecido adiposo, que não pode ser revertida completamente com a terapia nutricional convencional, acarretando assim o comprometimento funcional do organismo. Pacientes com neoplasias malignas apresentam marcadores inflamatórios elevados. Essa reação inflamatória sistêmica está relacionada à anorexia, à desnutrição e à caquexia, o que facilita a progressão do tumor e, consequentemente, aumenta a morbimortalidade ${ }^{21}$.

Segundo o INCA ${ }^{3}$, a síndrome da anorexia-caquexia em pacientes oncológicos é frequente e atinge aproximadamente $66,4 \%$ dos pacientes com perda de peso superior a $10 \%$. Em contrapartida, no presente estudo, a ausência de caquexia foi encontrada em 60,64\% da amostra analisada e 39,6\% apresentavam quadro de extrema desnutrição; entretanto, esse percentual é relevante, uma vez que a caquexia tem etiologia complexa e multifatorial, na qual a perda de peso progressiva e o IMC são apenas alguns dos componentes.
No presente estudo, 98,8\% dos pacientes apresentavam perda de peso no início da TNE; destes, $88,5 \%$ tiveram perda grave, e, de acordo com a classificação do IMC, 66,6\% estavam desnutridos, sendo a média de IMC antes da TNE 19,44 $4,83 \mathrm{Kg} / \mathrm{m}^{2}$. Dessa forma, a possível não consideração de aspectos clínicos, tais como a anorexia e o aumento da inflamação sistêmica, pode ter interferido na identificação da presença ou ausência da caquexia.

A terapia nutricional como adjuvante no tratamento dos pacientes oncológicos é de extrema importância. Para que esta seja efetiva, é recomendado que a nutrição enteral seja precoce, administrada de 24 a 48 horas após a admissão do paciente. As necessidades energéticas devem ser atendidas entre o terceiro e o sétimo dia de TNE, contribuindo para a recuperação do estado nutricional do paciente, aumentando a resposta ao tratamento antineoplásico e diminuindo seus efeitos adversos ${ }^{22}$.

Considerando os parâmetros antropométricos, após sete dias de TNE, a média de IMC foi de 18,76 $\pm 4,47 \mathrm{~kg} /$ $\mathrm{m}^{2}$, e $69,7 \%$ dos pacientes encontravam-se desnutridos, de acordo com a classificação do IMC. Em relação ao percentual de perda de peso, $52,1 \%$ dos pacientes continuaram a perder peso ( $46,9 \%$ perda grave) Entretanto, 47,9\% não apresentaram perda de peso após sete dias de TNE, sendo que $27,1 \%$ tiveram ganho de peso e $20,8 \%$ mantiveram o peso.

Comparando esses resultados com os obtidos no início da TNE, observou-se que houve uma redução de 47,9\% $(\mathrm{n}=46)$ no número de pacientes que apresentaram perda de peso, evidenciando o ganho ou a sua manutenção. Estudos demonstram que a TNE retarda ou impede a perda de peso em pacientes oncológicos. ${ }^{2}$ Corroborando esses estudos, Gevaerd et al..$^{23}$ constataram o ganho de peso em 45,2\% dos pacientes oncológicos avaliados e Gavazzi et al. ${ }^{24}$ observaram a manutençáo do peso em $48,7 \%$. Os autores afirmam que os pacientes em TNE apresentam maior chance de concluir o tratamento antineoplásico quando submetidos a essa terapia.

Entretanto, a TNE pode ser comprometida, dificultando o alcance do aporte calórico-proteico adequado, por questóes como procedimentos de rotina do hospital, atividades de rotina com o paciente, retirada e repassagem da sonda e intolerâncias gastrointestinais ${ }^{25}$. No presente estudo, acredita-se que esses fatores possam ter influenciado no fato de, após sete dias em TNE, 52,1\% $(\mathrm{n}=50)$ dos pacientes continuarem a perder peso.

\section{CONCLUSÃO}

Diante dos resultados obtidos, conclui-se que, no hospital em questão, o maior número de internações é de pacientes conveniados pelo SUS, do sexo masculino, 
idosos, com baixa escolaridade e, em sua maioria, apresentaram algum contato com álcool e tabaco. Nota-se maior prevalência de câncer de cabeça e pescoço em pacientes desnutridos ou com risco de desnutrição em razáo das complicaçôes da doença de base e do tratamento antineoplásico instituído.

Além disso, observou-se que a TNE contribuiu, de forma positiva, para o retardo da perda de peso desses pacientes que possuem um grande comprometimento do estado nutricional, consequentemente, favorecendo a resposta ao tratamento antineoplásico e diminuindo, possivelmente, o tempo de internação.

Os resultados sugerem a necessidade de uma intervenção nutricional precoce, uma vez que esses pacientes têm seu estado nutricional comprometido de forma progressiva. Ciente de que a TNE é capaz de reverter esse quadro, uma maior conscientizaçáo da sua utilização e benefícios é de suma importância, visto que essa terapia pode favorecer o prognóstico do paciente.

\section{CONTRIBUIÇÕES}

Michelle Martins Bortoletto e Iury Antônio de Souza participaram da concepçáo, aquisição e análise, interpretação dos dados da pesquisa e redação do trabalho. Anna Marcella Neves Dias participou do desenho do trabalho, análise e revisão crítica com contribuição social. Nilva Maria de Almeida participou do desenho do trabalho, aquisição, análise e revisão crítica com contribuição social. Elisa Grossi Mendonça participou da concepção, interpretação dos dados da pesquisa, revisão crítica com contribuição social e aprovação final do trabalho.

\section{DECLARAÇÃO DE CONFLITOS DE INTERESSE}

Nada a Declarar.

\section{REFERÊNCIAS}

1. Instituto Nacional de Câncer Alencar Gomes da Silva. O que é Câncer? [internet]. 2017 [acesso em 2017 jun 01]. Disponível em: http://www2.inca.gov.br/wps/wcm/ connect/cancer/site/oquee.

2. Cancer.org. American Cancer Society. Cancer Facts \& Figures 2016 [internet]. Atlanta, GA: American Cancer Society, Inc.;2016 [acesso em 2016 ago 15]. Disponível em: http://www.cancer.org/acs/groups/content/@ research/documents/ document/acspc-047079.pdf.

3. Instituto Nacional de Câncer José Alencar Gomes da Silva. Consenso Nacional de Nutrição Oncológica. Rio de Janeiro: INCA; 2015.

4. Nunes GR, Resende FR, Silva DCG. Análise comparativa do volume de dieta enteral prescrito com o volume de dieta infundido em pacientes internados em um hospital do município de Muriaé (MG). Rev Cient FAMINAS; 2015; 11(2):23-32

5. Kolankiewicz ACB, Souza MM, Magnago STBS, Domenico EBL. Apoio social percebido por pacientes oncológicos e sua relação com as características sociodemográficas. Rev Gaucha Enferm. 2015; 35(1):31-8.

6. Vieira EMM, Galvão ACP, Costa $\mathrm{HCB}$, Amorim ACL, Pinto JV, Ribeiro RGSP, et al. Perfil nutricional de pacientes oncológicos atendidos no hospital filantrópico do município de Cuiabá (MT). Arch Health Invest. 2014; 3(3):76-83.

7. Modena CM, Martins AB, Ribeiro RBN, Almeida SSL. Os homens e o adoecimento por câncer: um olhar sobre a produção científica brasileira. Rev Baia Sal Pub. 2013; 37(3):644-60.

8. Boaventura AP, Vedovato CA, Santos FF. Perfil dos pacientes oncológicos atendidos em uma unidade de emergência. Cienc y Enf. 2015; 21(2):51-62.

9. Howlader N, Noone AM, Krapcho M, Miller D, Bishop K, Ruhl J, et al. SEER Cancer Statistics Review, 19752014. National Cancer Institute. [internet] 2017 [acesso em 2017 set 21]. Disponível em: https://seer.cncer.gov/ csr/1975_2014/.

10. Le Campion ACOV, Santos KCB, Carmo ES, Silva FF, Peixoto FB, Ribeiro CMB, et al. Caracterização do atraso no diagnóstico do câncer de boca e orofaringe em dois centros de referência. Cad Saud Col. 2016; 24(2): 178-84.

11. Galbiatti ALS, Ruiz MT, Maniglia JV, Raposo LS, Pavarino-Bertelli EC, Goloni-Bertollo EM. Câncer de cabeça e pescoço: polimorfismos genéticos e metabolismo do folato. Rev Bras Otorrino. 2012; 1(78):132-39.

12. Brito LF, Silva LSS, Fernandes DD, Pires RA, Nogueira ADR, Souza CL, et al. Perfil nutricional de pacientes com câncer assistidos pela casa de acolhimento ao paciente oncológico do Sudoeste da Bahia. Rev Bras Can. 2012; 58(2):163-171.

13. Figueiredo RJ, Andrade RS, Pires DSM. Associação entre estado nutricional e qualidade de vida de pacientes com câncer de cabeça e pescoço. Rev Bras Cir Cabeça e Pescoço. 2016; 45(4):126-131.

14. Medeiras FPP, Martinez CE, Cardoso SS. Estado nutricional e ingestão alimentar do paciente com câncer de cabeça e pescoço submetidos a tratamento oncológico. Arq Bras Cien Saud. 2016; 23 (4):43-7.

15. Azevedo CD, Bosco SMD. Perfil nutricional de pacientes com câncer segundo diferentes indicadores de avaliaçáo. Rev Bras Jour.2012; 10(1):23-30.

16. Mouhayar E. Salahudeen A. Hypertension in Cancer Patients. Tex Heart Inst J. 2011; 38(3):263-65.

17. Pastore CA, Oehlschlaeger MHK, González MC. Impacto do estado nutricional e da força muscular sobre o estado de saúde geral e qualidade de vida em pacientes com câncer do trato gastrointestinal e pulmáo. Rev Bras Cancerol. 2013; 59(1): 43-9. 
18. Pressoir M, Desné S, Berchery D, Rossignol G, Poirre B, Meslier M, et al. Prevalence, risk factors and clinical implications of malnutrition in French Comprehensive Cancer Centers. Br J Cancer. 2010;102(6):966-97.

19. Cunha SFC, Tanaka LS, Salomão RG, Macedo DM, Santos TD, Peria FM. Nutrition screening in a university hospital: comparison between oncologic and nononcologic patients. Food and Nutriti Scienc. 2015; 13(6):75-82.

20. González MC, Borges LR, Silveira DH, Assunção MCF, Orlandi S. Validação da versão em português da avaliação subjetiva global produzida pelo próprio paciente. Rev Bras Nut Clin. 2010; 25(2):102-8.

21. Bozzetti F. Nutritional support in oncologic patients: where we are and where we are going. Clin Nutr. 2011; 30(6):714-7.

22. Society of Critical Care Medicine - SCCM; American Society for Parenteral and Essential Nutrition - ASPEN. Guidelines for the Provision and Assessment of Nutrition Support Therapy in the Adult Critically ill Patient. J of Parent and Enter Nutrition. 2009; 33(3):277-316.

23. Gevaerd SR, Fabre MES, Búrigo T, Carneiro CM, Pastore JA, Tarachuque CC, et al. Impacto da terapia nutricional enteral ambulatorial em pacientes oncológicos. Rev Bras Nut Clín. 2008; 23(1):41-5.

24. Gavazzi C, Colatruglio S, Valoriani F, Mazzaferro V, Sabbatini A, Biffi R, et al. Impacto fhome enteral nutrition in malnourished patients with upper gastrointestinal cancer: a multicentre randomised clinical trial. Eur J Cancer. 2016; 64 (9):107-12.

25. Ribeiro LMK, Oliveira Filho RS, Lima PA, Damasceno NRT, Soriano FG. Adequação dos balanços energético e proteico na nutrição por via enteral em terapia intensiva: quais são os fatores limitantes? Rev Bras Ter Intensiva. 2014;26(2):155-62. 